\title{
Crescimento radicular de soja em razão da sucessão de cultivos e da compactação do solo(1)
}

\begin{abstract}
Rosemeire Helena da Silva( ${ }^{(2)}$ e Ciro Antonio Rosolem ${ }^{(3)}$
Resumo - O objetivo do experimento foi avaliar o crescimento radicular e produção de matéria seca da parte aérea da soja (Glycine max (L.) Merrill) cultivada após diversas espécies vegetais, em solo com diferentes níveis de compactação. $\mathrm{O}$ trabalho foi realizado em vasos contendo amostras de um Latossolo Vermelho, textura franco arenosa, com camada de 3,5 $\mathrm{cm}$ (profundidade de 15 a 18,5 cm) compactada até as densidades $1,12,1,36$ e 1,60 $\mathrm{Mg} \mathrm{m}^{-3}$, onde cultivaram-se anteriormente aveia-preta, guandu, milheto, mucuna-preta, soja, sorgo granífero e tremoço-azul, e um tratamento sem planta (pousio). Essas espécies se desenvolveram por 37 a 39 dias, foram cortadas ao nível do solo, picadas em partes de aproximadamente $3 \mathrm{~cm}$ e deixadas sobre a superfície do vaso por 40 dias. Após esse período, cultivou-se a soja até 28 dias após a emergência, quando, então, as plantas foram colhidas. Foram avaliados produção de matéria seca da parte aérea e de raízes, e comprimento e diâmetro radicular da soja. O cultivo anterior com aveia-preta, guandu e milheto favoreceu o crescimento radicular da soja abaixo de camadas compactadas do solo. Independentemente do nível de compactação, o cultivo anterior com qualquer das espécies estudadas beneficiou a produção de matéria seca da parte aérea da soja.

Termos para indexação: Glycine max, rotação de culturas, matéria seca, densidade do solo.
\end{abstract}

\section{Soybean root growth as affected by previous crop and soil compaction}

\begin{abstract}
This study aimed at evaluating root growth and shoot dry matter production of soybean (Glycine max (L.) Merrill) cropped after different vegetal species, in a soil with different compaction levels. The experiment was conducted in pots containing a Dark-Red Latosol (Acrortox, loamy sand), and the pots had a layer $3.5 \mathrm{~cm}(15$ to $18.5 \mathrm{~cm})$ thick and $15 \mathrm{~cm}$ deep compacted to 1.12, 1.36 and $1.60 \mathrm{Mg} \mathrm{m}^{-3}$. Before soybean, the pots were cropped with black oat, pigeon pea, pearl millet, black mucuna, soybean, grain sorghum and lupin, plus a treatment without plants. These species were grown for 37 to 39 days, when they were cut at soil level, prick in particles of approximately $3 \mathrm{~cm}$ length, and left on the soil surface for 40 days. After this, soybean was planted in the pots and was allowed to grow for 28 days after plant emergence. The soybean shoot dry matter weight, root length, diameter and dry matter were evaluated. The previous crop with black oat, pigeon pea and pearl millet favored the soybean root growth below compacted layer soil. Regardless soil compaction, the soybean shoot dry matter was favored by the previous crop.
\end{abstract}

Index terms: Glycine max, crop rotation, dry matter, soil density.

(1) Aceito para publicação em 20 de agosto de 2001 . Extraído da dissertação de mestrado apresentada pelo primeiro autor à Universidade Estadual Paulista (Unesp), Faculdade de Ciências Agronômicas (FCA), Botucatu, SP. Financiado pela Fapesp.

(2) Unesp, FCA, Dep. de Produção Vegetal, Caixa Postal 237, CEP 18603-970 Botucatu, SP. Bolsista da Fapesp. E-mail: meire@fca.unesp.br

(3) Unesp, FCA, Dep. de Produção Vegetal.

E-mail: rosolem@fca.unesp.br

\section{Introdução}

Entre as conseqüências diretas da compactação do solo estão as reduções da porosidade e da infiltração de água, e aumento da resistência à penetração de raízes (Kirkegaard et al., 1993). Isso afeta a distribuição e morfologia das raízes. A compactação do solo pode causar redução de até $60 \%$ do crescimento radicular de algumas espécies (Bengough \& 
Mullins, 1991; Iijima et al., 1991; Panayiotopoulos et al., 1994). Em relação à soja, a resistência de $0,69 \mathrm{MPa}$ à penetração ocasionou redução de 50\% no crescimento radicular (Rosolem et al., 1994).

Entre as modificações morfológicas nas raízes, provocadas pela restrição ao crescimento, estão o aumento do diâmetro e a diminuição do comprimento, tornando-as tortuosas. Essas modificações, geralmente, são decorrentes do crescimento das raízes nos pontos de menor resistência oferecidos pelo solo (Borges et al., 1988), como canais deixados por raízes decompostas e fendas naturalmente encontradas no solo (Keisling et al., 1995). Assim, uma das estratégias para amenizar os efeitos da compactação pode ser o cultivo de espécies com sistema radicular vigoroso, que deixem canais que propiciem condições ao desenvolvimento de raízes da cultura subseqüente (Wang et al., 1986).

Desta forma, espécies de crescimento inicial rápido e agressivo, que proporcionam boa cobertura do solo (Nuernberg et al., 1986), com raízes capazes de penetrar em camadas com altas resistências à penetração (Materechera et al., 1992; Alvarenga et al., 1996), permitiriam melhor desenvolvimento da cultura sucessora.

O objetivo deste trabalho foi avaliar o crescimento radicular e produção de matéria seca da parte aérea da soja cultivada em vasos após sete espécies vegetais, em solo com diferentes estados de compactação.

\section{Material e Métodos}

O experimento foi realizado em casa de vegetação na Faculdade de Ciências Agronômicas/Unesp, Campus de Botucatu. A terra utilizada foi proveniente da camada arável $(0-20 \mathrm{~cm})$ de um Latossolo Vermelho, textura franco arenosa, com $680 \mathrm{~g} \mathrm{~kg}^{-1}$ de areia, $150 \mathrm{~g} \mathrm{~kg}^{-1}$ de silte e $160 \mathrm{~g} \mathrm{~kg}^{-1}$ de argila, cuja análise química mostrou $\mathrm{pH}$ em $\mathrm{CaCl}_{2}$ de 5,3, $3 \mathrm{mg} \mathrm{dm}^{-3}$ de P, 0,7, 13 e $9 \mathrm{mmol}_{\mathrm{c}} \mathrm{dm}^{-3}$ de $\mathrm{K}$, $\mathrm{Ca}$ e $\mathrm{Mg}$, respectivamente, e saturação por bases de $51 \%$. O preparo da terra constou das seguintes etapas: peneiramento em malha de $4 \mathrm{~mm}$; calagem com $0,650 \mathrm{~g}$ de calcário por vaso , com PRNT 100\% (32\% de CaO e 18\% $\mathrm{MgO}$ ), para elevar a saturação por bases a $70 \%$; e adubação com 150, 120, 1,0 e 0,5 $\mathrm{mg} \mathrm{dm}^{-3}$ de P, K, Zn e B, utilizando como fontes superfosfato simples, cloreto de potássio, sulfato de zinco e bórax, respectivamente.
O experimento foi instalado em esquema fatorial $3 \times 8$, inteiramente casualizado, com quatro repetições. Os tratamentos constaram do cultivo de aveia-preta (Avena strigosa) cv. Comum; guandu (Cajanus cajan) cv. Fava Larga; milheto (Pennisetum americanum); mucuna-preta (Stizolobium aterrimum); soja (Glycine max) cv. FT Estrela; sorgo (Sorgum bicolor) Híbrido A-6304; e tremoço-azul (Lupinus angustifolium) cv. IAPAR 24; e de um tratamento sem planta (pousio), em solo com densidades de 1,12, 1,36 e $1,60 \mathrm{Mg} \mathrm{m}^{-3}$.

Os vasos foram constituídos pela sobreposição de três anéis de PVC, de $10 \mathrm{~cm}$ de diâmetro interno: o superior e o inferior, com $15 \mathrm{~cm}$ de altura cada um, receberam solo com densidade de $1,12 \mathrm{Mg} \mathrm{m}^{-3}$, e o intermediário, com $3,5 \mathrm{~cm}$ de altura, recebeu os tratamentos de compactação $(1,12$, 1,36 e $1,60 \mathrm{Mg} \mathrm{m}^{-3}$ ). Na obtenção dos níveis de compactação, foram adicionadas quantidades determinadas de solo, com $8 \%$ de umidade ( $80 \%$ da retenção de água do vaso), ao anel de volume conhecido, e em seguida este anel sofreu golpes sucessivos de uma massa de ferro até atingir os valores de densidade do solo desejados.

Após a montagem, os vasos foram pesados com a finalidade de manter a umidade inicial durante a realização do experimento. Em seguida, foram semeadas as sete espécies de plantas, fazendo-se o controle da umidade por pesagens diárias e regas pela superfície. Nos vasos correspondentes aos tratamentos, cultivou-se uma planta de guandu, mucuna-preta, soja e tremoço-azul, duas plantas de sorgo, e três plantas de aveia-preta e milheto. As populações foram definidas numa tentativa de simular as proporções com as populações de cada espécie no campo. Aos 18 dias após a emergência das plantas, aplicaramse, em cobertura, $50 \mathrm{mg} \mathrm{dm}^{-3}$ de $\mathrm{N}$ (sulfato de amônio). Aos 37 a 39 dias após a emergência, as plantas foram cortadas na altura do solo, picadas em partes com aproximadamente $3 \mathrm{~cm}$, e deixadas na superfície dos vasos por 40 dias. Nesse intervalo, os vasos foram mantidos com umidade a $80 \%$ da capacidade de retenção de água para facilitar a decomposição das raízes.

Após esse período, a soja foi semeada com sementes pré-germinadas durante três dias, em rolo de papel, sem nenhuma adubação adicional. Dois dias após a emergência, fez-se o desbaste, deixando-se uma planta por vaso. A colheita da soja se deu aos 28 dias após a semeadura, e foram feitas avaliações de produção de matéria seca da parte aérea e das raízes em cada uma das camadas do vaso, e do comprimento e diâmetro radicular. Para essas avaliações, os vasos foram desmontados, considerando-se separadamente cada uma das três camadas de solo, e as raízes foram separadas da terra por lavagem sobre peneira de $0,5 \mathrm{~mm}$ de malha, separando-se os debris manualmente. $\mathrm{O}$ comprimento radicular foi determinado pelo método de 
Tennant (1975), e o diâmetro de acordo com Hallmark \& Barber (1984).

Os resultados obtidos foram submetidos à análise de variância e as médias comparadas pelo teste DMS a 5\% de probabilidade.

\section{Resultados e Discussão}

Houve interação das espécies de cobertura com as densidades do solo na produção de matéria seca de raízes da soja, tanto total como por camadas, e no comprimento e diâmetro radicular nas camadas central e inferior dos vasos (Tabela 1). Em relação ao comprimento radicular total e na camada superior do vaso, houve efeito de espécies de cobertura e de densidades do solo, mas não da interação entre eles, e quanto ao diâmetro das raízes nesta camada e matéria seca da parte aérea da soja, houve apenas o efeito das espécies cultivadas antes da soja.

$\mathrm{O}$ aumento da densidade do solo na camada central do vaso (15 a 18,5 cm) não afetou o peso da matéria seca das raízes da soja na camada superior dos vasos (0 a $15 \mathrm{~cm})$, exceto quando cultivada após a aveia-preta, observando-se redução dos valores nas densidades de 1,36 e 1,60 $\mathrm{Mg} \mathrm{m}^{-3}$ (Tabela 2). Quando se compararam os resultados ora obtidos com os constatados a campo (Iijima et al., 1991) ou em vasos (Silva et al., 1992; Rosolem et al., 1994), era esperado maior desenvolvimento superficial de raízes nas maiores densidades, o que não foi observado no presente trabalho. Em geral, os resultados não mostraram influência do aumento na densidade do solo sobre o desenvolvimento de raízes da soja nesta camada. Resultados semelhantes foram obtidos por Rosolem et al. (1994), Fernandez et al. (1995) e Alvarenga et al. (1996).

A compactação do solo teve maior efeito sobre a produção de matéria seca de raízes da soja na camada central, exceto quando cultivada após tremoçoazul e pousio, e na inferior $(18,5$ a 33,5 cm) (Tabela 2). Esperava-se que após o pousio o desenvolvimento das raízes fosse menor, pois, conforme Wang et al. (1986), o crescimento das raízes de soja depende de canais preexistentes nos primeiros $35 \mathrm{a} 40 \mathrm{~cm}$, que são resultados de raízes mortas. No entanto, no presente trabalho, o efeito da compactação do solo na produção de matéria seca de raízes da soja após o pousio foi semelhante ao observado após as outras espécies, não se verificando influência direta de canais deixados no cultivo anterior (Tabela 2).

$\mathrm{O}$ aumento da densidade do solo causou redução de $30 \%$ a $35 \%, 22 \%$ a $24 \%$ e de $21 \%$ a $26 \%$ da matéria seca total de raízes, quando a soja foi cultivada após aveia-preta, guandu e milheto, respectivamente (Tabela 2). Essas reduções foram menores que as constatadas por Rosolem et al. (1994), quando a redução no crescimento radicular da soja foi de $50 \%$.

Mesmo que o crescimento radicular, expresso pela produção de matéria seca nas camadas central e inferior, após aveia-preta, guandu e milheto tenha sido afetado pela compactação, verifica-se que a produção de matéria seca total da soja após o guandu, nos três níveis de densidade, e após aveia-preta, nas duas menores densidades, em média, foi maior que após as outras espécies (Tabela 2). Com o aumento na densidade do solo, os efeitos dessas espécies desapareceram, indicando que a resistência à penetração se torna o fator mais limitante. Portanto, os benefícios dos bioporos no crescimento de raízes (Ehlers et al., 1983; Wang et al., 1986) dependem do nível de compactação.

Ao contrário da produção de matéria seca, o comprimento de raízes da soja na camada superior dos vasos aumentou com o aumento da densidade do

Tabela 1. Análise de variância dos valores de matéria seca da parte aérea, e matéria seca, comprimento e diâmetro de raízes da soja em razão da compactação do solo.

\begin{tabular}{|c|c|c|c|c|}
\hline \multirow[t]{2}{*}{ Camada do solo } & \multicolumn{3}{|c|}{ Causas de variação } & \multirow[t]{2}{*}{$\mathrm{CV}(\%)$} \\
\hline & $\begin{array}{c}\text { Espécies } \\
\text { (E) }\end{array}$ & $\begin{array}{c}\text { Densidades } \\
\text { (D) }\end{array}$ & ExD & \\
\hline & \multicolumn{4}{|c|}{ Matéria seca da parte aérea } \\
\hline & ** & & & 15,57 \\
\hline & \multicolumn{4}{|c|}{ Matéria seca de raízes } \\
\hline Total & ** & & & 21,55 \\
\hline Camada superior & ** & ns & * & 24,39 \\
\hline Camada central & $*$ & $* *$ & ** & 39,92 \\
\hline \multirow[t]{2}{*}{ Camada inferior } & ** & & & 46,16 \\
\hline & \multicolumn{4}{|c|}{ Comprimento das raízes } \\
\hline Total & ** & ** & ns & 21,35 \\
\hline Camada superior & ** & * & ns & 18,10 \\
\hline Camada central & ns & ** & ** & 39,15 \\
\hline \multirow[t]{2}{*}{ Camada inferior } & ** & ** & ** & 70,65 \\
\hline & \multicolumn{4}{|c|}{ Diâmetro das raízes } \\
\hline Camada superior & $* *$ & ns & ns & 8,51 \\
\hline Camada central & ** & $* *$ & ** & 11,99 \\
\hline Camada inferior & * & $* *$ & * & 82,07 \\
\hline
\end{tabular}

Pesq. agropec. bras., Brasília, v. 37, n. 6, p. 855-860, jun. 2002 
Tabela 2. Matéria seca de raízes da soja ( $\mathrm{g}$ por vaso) nas camadas superior, central e inferior dos vasos e matéria seca total em razão do cultivo anterior e das densidades do solo ${ }^{(1)}$.

\begin{tabular}{|c|c|c|c|c|c|c|c|c|}
\hline \multirow{2}{*}{$\begin{array}{l}\text { Densidades } \\
\text { do solo } \\
\left(\mathrm{Mg} \mathrm{m}^{-3}\right)\end{array}$} & \multicolumn{8}{|c|}{ Cultivo anterior } \\
\hline & Aveia-preta & Guandu & Milheto & Mucuna-preta & Soja & Sorgo & Tremoço-azul & Pousio \\
\hline & \multicolumn{8}{|c|}{ Camada superior } \\
\hline 1,12 & $0,74 \mathrm{aA}$ & $0,59 \mathrm{aB}$ & $0,49 \mathrm{aB}$ & $0,24 \mathrm{aC}$ & $0,33 \mathrm{aC}$ & $0,30 \mathrm{aC}$ & $0,35 \mathrm{aC}$ & $0,22 \mathrm{aC}$ \\
\hline 1,36 & $0,52 \mathrm{bAB}$ & $0,56 \mathrm{aA}$ & $0,38 \mathrm{aBC}$ & $0,32 \mathrm{aC}$ & $0,31 \mathrm{aC}$ & $0,25 \mathrm{aC}$ & $0,36 \mathrm{aC}$ & $0,30 \mathrm{aC}$ \\
\hline \multirow[t]{2}{*}{1,60} & $0,52 \mathrm{bAB}$ & $0,53 \mathrm{aA}$ & $0,44 \mathrm{aABCD}$ & $0,30 \mathrm{aD}$ & $0,37 \mathrm{aCD}$ & $0,39 \mathrm{aCD}$ & $0,46 \mathrm{aABC}$ & $0,34 \mathrm{aCD}$ \\
\hline & \multicolumn{8}{|c|}{ Camada central } \\
\hline 1,12 & $0,05 \mathrm{aA}$ & $0,05 \mathrm{aA}$ & $0,04 \mathrm{aAB}$ & $0,02 \mathrm{bC}$ & $0,03 \mathrm{abBC}$ & $0,04 \mathrm{abAB}$ & $0,03 \mathrm{aBC}$ & $0,03 \mathrm{aBC}$ \\
\hline 1,36 & $0,04 \mathrm{abAB}$ & $0,01 \mathrm{cC}$ & $0,04 \mathrm{aB}$ & $0,04 \mathrm{aAB}$ & $0,04 \mathrm{aA}$ & $0,05 \mathrm{aA}$ & $0,03 \mathrm{aAB}$ & $0,02 \mathrm{aBC}$ \\
\hline \multirow[t]{2}{*}{1,60} & $0,02 \mathrm{bAB}$ & $0,03 \mathrm{bA}$ & $0,02 \mathrm{bAB}$ & $0,01 \mathrm{bB}$ & $0,02 \mathrm{bAB}$ & $0,02 \mathrm{bAB}$ & $0,02 \mathrm{aAB}$ & $0,01 \mathrm{aAB}$ \\
\hline & \multicolumn{8}{|c|}{ Camada inferior } \\
\hline 1,12 & $0,05 \mathrm{aB}$ & $0,10 \mathrm{aA}$ & $0,05 \mathrm{aB}$ & $0,04 \mathrm{aB}$ & $0,10 \mathrm{aA}$ & $0,05 \mathrm{aB}$ & $0,05 \mathrm{aB}$ & $0,09 \mathrm{aA}$ \\
\hline 1,36 & $0,03 \mathrm{abBCD}$ & $0,01 \mathrm{bD}$ & $0,01 \mathrm{bCD}$ & $0,03 \mathrm{abBCD}$ & $0,04 \mathrm{bAB}$ & $0,05 \mathrm{aA}$ & $0,04 \mathrm{aABC}$ & $0,04 \mathrm{bAB}$ \\
\hline \multirow[t]{2}{*}{1,60} & $0,01 \mathrm{bA}$ & $0,01 \mathrm{bA}$ & $0,01 \mathrm{bA}$ & $0,00 \mathrm{bA}$ & $0,00 \mathrm{cA}$ & $0,00 \mathrm{bA}$ & $0,00 \mathrm{bA}$ & $0,00 \mathrm{cA}$ \\
\hline & \multicolumn{8}{|c|}{ Matéria seca total } \\
\hline 1,12 & $0,84 \mathrm{aA}$ & $0,74 \mathrm{aA}$ & $0,58 \mathrm{aB}$ & $0,30 \mathrm{aD}$ & $0,46 \mathrm{aBC}$ & $0,39 \mathrm{aCD}$ & $0,42 \mathrm{aCD}$ & $0,43 \mathrm{aCD}$ \\
\hline 1,36 & $0,58 \mathrm{bA}$ & $0,58 \mathrm{bA}$ & $0,43 \mathrm{bB}$ & $0,40 \mathrm{aB}$ & $0,40 \mathrm{aB}$ & $0,36 \mathrm{aB}$ & $0,42 \mathrm{aB}$ & $0,37 \mathrm{aB}$ \\
\hline 1,60 & $0,55 \mathrm{bAB}$ & $0,56 \mathrm{bA}$ & $0,46 \mathrm{abABC}$ & $0,32 \mathrm{aD}$ & $0,32 \mathrm{aD}$ & $0,42 \mathrm{aBCD}$ & $0,49 \mathrm{aABC}$ & $0,36 \mathrm{aCD}$ \\
\hline
\end{tabular}

solo na camada subsuperficial, porém sem efeito no diâmetro. O comprimento total, por sua vez, diminuiu (Tabela 3). Este resultado mostra que, independentemente do cultivo anterior, o alongamento das raízes da soja foi limitado pelo impedimento mecânico do solo, concordando com os resultados de Borges et al. (1988), Johnson et al. (1990), Rosolem et al. (1994) e Fernandez et al. (1995).

No geral, essas características sofreram efeito do cultivo anterior e do pousio, podendo-se constatar que a soja após o guandu teve o maior comprimento de raízes na camada superior, não diferindo apenas do tratamento após o tremoço-azul, e o diâmetro foi maior quando a soja foi cultivada após a aveia-preta (Tabela 3). Em relação ao comprimento total de raízes, a soja apresentou maior valor após o guandu, não diferindo do tremoço-azul e do pousio. Tal resultado não pode ser atribuído à presença de canais no solo nesta camada, pois foi observado também no pousio, mas nota-se que a importância dos canais deixados por raízes do cultivo anterior é maior quando existentes na camada compactada e não na camada acima dela.

Em solos compactados, geralmente ocorre redução do comprimento de raízes associada ao aumento do diâmetro em decorrência das restrições ao cres-
Tabela 3. Características de raízes da soja em razão das espécies e das densidades do solo ${ }^{(1)}$.

\begin{tabular}{lccc}
\hline Cultivo anterior & $\begin{array}{c}\text { Comprimento } \\
\text { total }\end{array}$ & $\begin{array}{c}\text { Comprimento } \\
\text { na camada } \\
\text { superior }\end{array}$ & $\begin{array}{c}\text { Diâmetro na } \\
\text { camada superior }\end{array}$ \\
\hline Aveia-preta & $-------(m$ por vaso)---------- & ( $\mu$ m por vaso) \\
Guandu & $4,83 \mathrm{c}$ & $3,54 \mathrm{~cd}$ & $46 \mathrm{a}$ \\
Milheto & $6,10 \mathrm{a}$ & $4,30 \mathrm{a}$ & $41 \mathrm{bc}$ \\
Mucuna-preta & $4,86 \mathrm{c}$ & $3,19 \mathrm{~cd}$ & $40 \mathrm{bcd}$ \\
Soja & $4,74 \mathrm{c}$ & $2,48 \mathrm{e}$ & $40 \mathrm{bcd}$ \\
Sorgo & $5,16 \mathrm{bc}$ & $2,71 \mathrm{e}$ & $42 \mathrm{~b}$ \\
Tremoço-azul & $4,76 \mathrm{c}$ & $2,53 \mathrm{e}$ & $39 \mathrm{~cd}$ \\
Pousio & $5,95 \mathrm{ab}$ & $3,93 \mathrm{ab}$ & $38 \mathrm{~d}$ \\
\hline Densidades $\left(\mathrm{Mg} \mathrm{m}^{-3}\right)$ & $5,58 \mathrm{abc}$ & $2,82 \mathrm{de}$ & $34 \mathrm{e}$ \\
1,12 & & & \\
1,36 & $66,26 \mathrm{a}$ & $30,02 \mathrm{~b}$ & $24 \mathrm{a}$ \\
1,60 & $51,12 \mathrm{~b}$ & $31,40 \mathrm{ab}$ & $24 \mathrm{a}$ \\
\hline
\end{tabular}

(1)Médias seguidas de letras iguais não diferem entre si pelo teste DMS, a $5 \%$ de probabilidade.

cimento (Borges et al., 1988; Fernandez, 1995). No presente trabalho, na camada central, tal efeito não foi comum. Porém, na camada inferior isso ocorreu nitidamente quando a soja foi cultivada após aveia-preta, guandu e milheto, principalmente na densidade de $1,60 \mathrm{Mg} \mathrm{m}^{-3}$ (Tabela 4). Masle \& Farquhar (1988) e Rosolem et al. (1994) sugeriram que o menor desenvolvimento das raízes após atravessarem a camada compactada ocorre porque elas não conseguem se recuperar do estresse ao qual foram 
Tabela 4. Comprimento e diâmetro de raízes de soja, na camada central e inferior dos vasos, em razão do cultivo anterior e das densidades do solo ${ }^{(1)}$.

\begin{tabular}{|c|c|c|c|c|c|c|c|c|}
\hline \multirow{2}{*}{$\begin{array}{l}\text { Densidades } \\
\text { do solo } \\
\left(\mathrm{Mg} \mathrm{m}^{-3}\right)\end{array}$} & \multicolumn{8}{|c|}{ Cultivo anterior } \\
\hline & Aveia-preta & Guandu & Milheto & Mucuna-preta & Soja & Sorgo & Tremoço-azul & Pousio \\
\hline & \multicolumn{8}{|c|}{ Comprimento (m por vaso) - Camada central } \\
\hline 1,12 & $1,27 \mathrm{aBCD}$ & $1,53 \mathrm{aBC}$ & $1,57 \mathrm{aB}$ & $2,51 \mathrm{aA}$ & $1,02 \mathrm{aCD}$ & $0,97 \mathrm{aD}$ & $0,95 \mathrm{bD}$ & $1,11 \mathrm{aBCD}$ \\
\hline 1,36 & $0,50 \mathrm{bCD}$ & $0,42 \mathrm{bD}$ & $1,01 \mathrm{bBC}$ & $0,92 \mathrm{bBCD}$ & $1,00 \mathrm{aBC}$ & $1,07 \mathrm{aAB}$ & $1,59 \mathrm{aA}$ & $1,00 \mathrm{aBC}$ \\
\hline \multirow[t]{2}{*}{1,60} & $0,60 \mathrm{bAB}$ & $0,79 \mathrm{bA}$ & $0,54 \mathrm{bAB}$ & $0,25 \mathrm{cB}$ & $0,47 \mathrm{bAB}$ & $0,73 \mathrm{aAB}$ & $0,70 \mathrm{bAB}$ & $0,34 \mathrm{bAB}$ \\
\hline & \multicolumn{8}{|c|}{ Diâmetro ( $\mu$ m por vaso) - Camada central } \\
\hline 1,12 & $33 \mathrm{bA}$ & $29 \mathrm{aA}$ & $27 \mathrm{bA}$ & $14 \mathrm{cB}$ & $28 \mathrm{abA}$ & $30 \mathrm{aA}$ & $31 \mathrm{aA}$ & $17 \mathrm{bB}$ \\
\hline 1,36 & $41 \mathrm{aA}$ & $28 \mathrm{aC}$ & $32 \mathrm{abBC}$ & $35 \mathrm{aB}$ & $33 \mathrm{aB}$ & $31 \mathrm{aBC}$ & $27 \mathrm{aC}$ & $19 \mathrm{abD}$ \\
\hline \multirow[t]{2}{*}{1,60} & $35 \mathrm{bA}$ & $31 \mathrm{aABC}$ & $32 \mathrm{aAB}$ & $29 \mathrm{bBCD}$ & $26 \mathrm{bCD}$ & $29 \mathrm{aBCD}$ & $28 \mathrm{aBCD}$ & $24 \mathrm{aD}$ \\
\hline & \multicolumn{8}{|c|}{ Comprimento (m por vaso) - Camada inferior } \\
\hline 1,12 & $1,04 \mathrm{aD}$ & $2,46 \mathrm{aBC}$ & $1,56 \mathrm{aCD}$ & $1,85 \mathrm{aCD}$ & $3,33 \mathrm{aAB}$ & $1,94 \mathrm{aCD}$ & $1,62 \mathrm{aCD}$ & $4,26 \mathrm{aA}$ \\
\hline 1,36 & $0,47 \mathrm{aBCD}$ & $0,02 \mathrm{bD}$ & $0,31 \mathrm{bCD}$ & $1,24 \mathrm{aABC}$ & $1,52 \mathrm{bAB}$ & $1,96 \mathrm{aA}$ & $1,20 \mathrm{aABC}$ & $1,55 \mathrm{bAB}$ \\
\hline 1,60 & $0,01 \mathrm{bA}$ & $0,16 \mathrm{bA}$ & $0,02 \mathrm{bA}$ & $0,00 \mathrm{bA}$ & $0,00 \mathrm{cA}$ & $0,00 \mathrm{bA}$ & $0,00 \mathrm{bA}$ & $0,00 \mathrm{cA}$ \\
\hline \multicolumn{9}{|c|}{ Diâmetro ( $\mu \mathrm{m}$ por vaso) - Camada inferior } \\
\hline 1,12 & $44 \mathrm{bA}$ & $34 \mathrm{bA}$ & $36 \mathrm{bA}$ & $28 \mathrm{bA}$ & $33 \mathrm{bA}$ & $27 \mathrm{bA}$ & $30 \mathrm{bA}$ & $22 \mathrm{bA}$ \\
\hline 1,36 & $137 \mathrm{bB}$ & $289 \mathrm{aA}$ & $62 \mathrm{bB}$ & $34 \mathrm{bB}$ & $34 \mathrm{bB}$ & $31 \mathrm{bB}$ & $32 \mathrm{bB}$ & $30 \mathrm{bB}$ \\
\hline 1,60 & $322 \mathrm{aB}$ & $254 \mathrm{aB}$ & $511 \mathrm{aA}$ & $0 \mathrm{aB}$ & $0 \mathrm{aB}$ & $0 \mathrm{aB}$ & $0 \mathrm{aB}$ & $0 \mathrm{aB}$ \\
\hline
\end{tabular}

submetidas, e que esse esforço provoca maior gasto de fotoassimilados. Esta pode ser a razão do menor comprimento radicular da soja na camada abaixo da compactada verificado no presente trabalho.

Fernandez et al. (1995) observaram que o crescimento das raízes de soja foi completamente inibido a partir da densidade de $1,52 \mathrm{Mg} \mathrm{m}^{-3}$. A capacidade das raízes em penetrar na camada compactada na densidade de $1,60 \mathrm{Mg} \mathrm{m}^{-3}$ após o cultivo da aveiapreta, guandu e milheto (Tabela 4) permite inferir que o cultivo dessas espécies pode promover a descompactação do solo por causa dos canais deixados pelas raízes dessas espécies após a decomposição, concordando com a hipótese levantada por Wang et al. (1986) e Unger \& Kaspar (1994).

A produção de matéria seca da parte aérea da soja cultivada após qualquer uma das espécies foi maior do que quando cultivada após o pousio, demonstrando resposta positiva do desenvolvimento inicial da soja a partir do cultivo em sucessão a outras espécies (Tabela 5). A soja cultivada após o tremoçoazul apresentou valor médio de matéria seca da parte aérea significativamente superior ao da soja plantada após milheto, soja e sorgo.

A falta de efeito da densidade do solo na produção de matéria seca da parte aérea da soja também foi
Tabela 5. Médias de matéria seca da parte aérea da soja em razão das espécies anteriores e das densidades do solo ${ }^{(1)}$.

\begin{tabular}{lc}
\hline Cultivo anterior & $\begin{array}{c}\text { Matéria seca da } \\
\text { parte aérea (g por vaso) }\end{array}$ \\
\hline Aveia-preta & $0,95 \mathrm{abc}$ \\
Guandu & $1,02 \mathrm{ab}$ \\
Milheto & $0,86 \mathrm{~cd}$ \\
Mucuna-preta & $0,95 \mathrm{abc}$ \\
Soja & $0,93 \mathrm{bcd}$ \\
Sorgo & $0,82 \mathrm{~d}$ \\
Tremoço-azul & $1,05 \mathrm{a}$ \\
Pousio & $0,66 \mathrm{e}$ \\
\hline Densidades $\left(\mathrm{Mg} \mathrm{m}^{-3}\right)$ & \\
1,12 & $0,95 \mathrm{a}$ \\
1,36 & $0,88 \mathrm{a}$ \\
1,60 & $0,89 \mathrm{a}$ \\
\hline
\end{tabular}

${ }^{(1)}$ Médias seguidas de letras iguais não diferem entre si pelo teste DMS, a $5 \%$ de probabilidade.

encontrada por Rosolem et al. (1994) e por Fernandez et al. (1995), mostrando ser este um resultado comum em experimentos de curta duração. Por sua vez, o efeito da compactação do solo sobre a produção de matéria seca da parte aérea das plantas é controverso, pois, conforme Atwell (1990), somente haverá prejuízo se existir deficiência de nutrientes ou água. Assim, em experimentos em vasos, não faltando água 
e nutrientes, é esperado menor efeito da compactação na produção de matéria seca da parte aérea.

\section{Conclusões}

1. O cultivo anterior com aveia-preta, guandu e milheto favorece o crescimento radicular da soja abaixo de camadas compactadas do solo.

2. Independentemente da compactação do solo, o cultivo anterior com qualquer uma das espécies estudadas beneficia a produção de matéria seca da parte aérea da soja.

\section{Referências}

ALVARENGA, R. C.; COSTA, L. M.; MOURA FILHO, W.; REGAZZI, A. J. Crescimento de leguminosas em camadas de solo compactadas artificialmente. Revista Brasileira de Ciência do Solo, Campinas, v. 20, p. 319 326, 1996.

ATWELL, B. J. The effect of soil compaction on wheat during early tillering. I. Growth, development and root structure. New Phytologist, Cambridge, Inglaterra, v. 115, p. 29-35, 1990.

BENGOUGH, A. G.; MULLINS, C. E. Penetrometer resistance, root penetration resistance and root elongation rate in two sandy loam soils. Plant and Soil, Dordrecht, v. 131, p. $59-66,1991$.

BORGES, E. N.; NOVAIS, R. F.; FERNANDEZ, B.; BARROS, N. F. Respostas de variedades de soja à compactação de camadas de solo. Revista Ceres, Viçosa, v. 35 , p. $553-568,1988$.

EHLERS, W.; RÜPRE, U.; HESSE, F.; BÖHM, W Penetration resistance and root growth of oats in tilled and untilled loess soil. Soil and Tillage Research, Amsterdam, v. 3, p. 261-275, 1983.

FERNANDEZ, E. M.; CRUSCIOL, C. A. C.; THIMOTHEO, C. M. S.; ROSOLEM, C. A. Matéria seca e nutrição da soja em razão da compactação do solo e adubação fosfatada. Científica, São Paulo, v. 23, n. 1, p. 117-132, 1995.

HALLMARK, W. B.; BARBER, S. A. Root growth and morphology, nutrient uptake and nutrient status of early growth of soybeans as affected by soil P and K. Agronomy Journal, Madison, v. 76, p. 209-212, 1984.

IIJIMA, M.; KONO, Y.; YAMAUCHI, A.; PARDALES JUNIOR, J. R. Effects of soil compaction on the development of rice and maize root systems. Environmental and Experimental Botany, Oxford, v. 31, n. 3, p. 333-342, 1991.
JOHNSON, J. F.; VOORHEES, W. B.; NELSON, W. W.; RANDALL, G. W. Soybean growth and yield as affected by surface and subsoil compaction. Agronomy Journal, Madison, v. 82, p. 973-979, 1990.

KEISLING, T.; BATCHELOR, J. T.; PORTER, O. A. Soybean root morphology in soils with and without tillage pans in the lower Mississippi River Valley. Journal of Plant Nutrition, New York, v. 18, n. 3, p. 373-384, 1995.

KIRKEGAARD, J. A.; SO, H. B.; TROEDSON, R. J. Effect of compaction on the growth of pigeon pea on clays soils. III. Effect of soil type and water regime on plant response. Soil and Tillage Research, Amsterdam, v. 26, p. 163-178, 1993.

MASLE, J.; FARQUHAR, G. D. Effects of soil strength on the relation of water-use efficiency and growth to carbon isotope discrimination in wheat seedlings. Plant Physiology, Rockville, v. 86, p. 32-38, 1988.

MATERECHERA, S. A.; ALSTON, A. M.; KIRBY, J. M.; DEXTER, A. R. Influence of root diameter on the penetration of seminal roots into a compacted subsoil. Plant and Soil, Dordrecht, v. 144, n. 2, p. 297-303, 1992.

NUERNBERG, N. J.; STAMMEL, J. G.; CABEDA, M. S. V. Efeito de sucessão de culturas e tipos de adubação em características físicas de um solo da encosta basáltica sul-rio-grandense. Revista Brasileira de Ciência do Solo, Campinas, v. 10, p. 185-190, 1986.

PANAYIOTOPOULOS, K. P.; PAPADOPOULOU, C. P.; HATJIIOANNIDOU, A. Compaction and penetration resistance of an Alfisol and Entisol and their influence on root growth of maize seedlings. Soil and Tillage Research, Amsterdam, v. 31, n. 4, p. 323-337, 1994.

ROSOlEM, C. A.; ALMEIDA, A. C. S.; SACRAMENTO, L. V. S. Sistema radicular e nutrição da soja em função da compactação do solo. Bragantia, Campinas, v. 53, n. 2, p. 259-266, 1994.

SILVA, G. P.; NOVAIS, R. F.; NEVES, J. C. L.; BARROS, N. F. Respostas de espécies de gramíneas forrageiras a camadas compactadas de solo. Revista Ceres, Viçosa, v. 39, p. 31-43, 1992.

TENNANT, D. A. A test of a modified line intersect method of estimating root length. Journal of Ecology, Oxford, v. 63, p. 995-1001, 1975.

UNGER, P. N.; KASPAR, J. C. Soil compaction and root growth: a review. Agronomy Journal, Madison, v. 86, p. 759-766, 1994.

WANG, J.; HESKETH, J. D.; WOOLLEY, J. T. Preexisting channels and soybean rooting patterns. Soil Science, Baltimore, v. 141, p. 432-437, 1986. 